\title{
Awake sedation with propofol during carotid endarterectomy provides better stress relief compared to alprazolam premedication alone
}

\author{
P. Szabó, G. Menyhei, Z. Horváth-Szalai, T. Kőszegi, G. Fazekas, T. Molnár \\ University of Pécs, Medical School, Hungary
}

Objectives: Carotid endarterectomy (CEA) in regional anaesthesia Increased stress - premedication or premed+sedation?

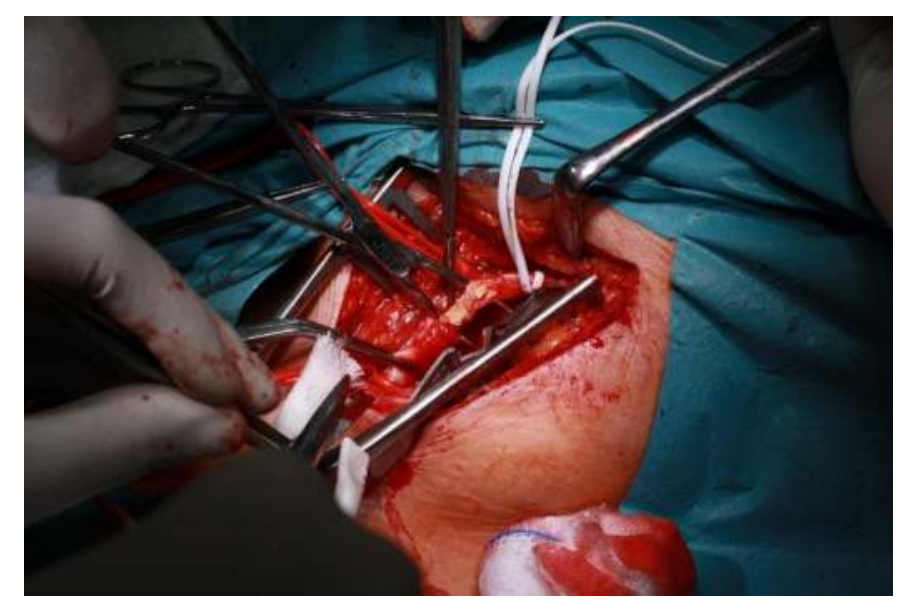

Table 1. Patients' demographic and clinical characteristics

\begin{tabular}{l|c|c|c} 
& TCI & BDZ & $\Sigma$ \\
\hline $\mathrm{N}$ & 20 & 20 & 40 \\
\hline shunt & 1 & 3 & 4 \\
\hline age & $62.65 \pm 8.71$ & $68.47 \pm 7.88$ & $65.32 \pm 8.74$ \\
\hline male & 14 & 17 & 31 \\
\hline female & 6 & 3 & 9 \\
\hline BMI & $27.37 \pm 5.8$ & $27.07 \pm 3.43$ & $27.24 \pm 4.83$ \\
\hline right sided operation & 7 & 11 & 18 \\
\hline left sided operation & 13 & 9 & 22 \\
\hline operated stenosis $(\%)$ & $84.75 \pm 5.73$ & $84.41 \pm 6.35$ & $84.59 \pm 5.94$ \\
\hline contralat. stenosis(\%) & $40.28 \pm 20.54$ & $49 \pm 27.53$ & $44.24 \pm 23.98$ \\
\hline clamping time (min) & $21.05 \pm 5.31$ & $20.19 \pm 6.57$ & $20.65 \pm 5.85$
\end{tabular}

\section{Methods:}

1. premedication $(0.25 \mathrm{mg}$ alprazolam per oral) was used for all subjects

2. cervical plexus block

3. randomization:

- intraop propofol sedation (TCI group) Schnider-protocoll, Ce=0,5-1 ug $\mathrm{ml}^{-1}$, Target: Ramsay 2-3 level

- control - only premed (BDZ group)
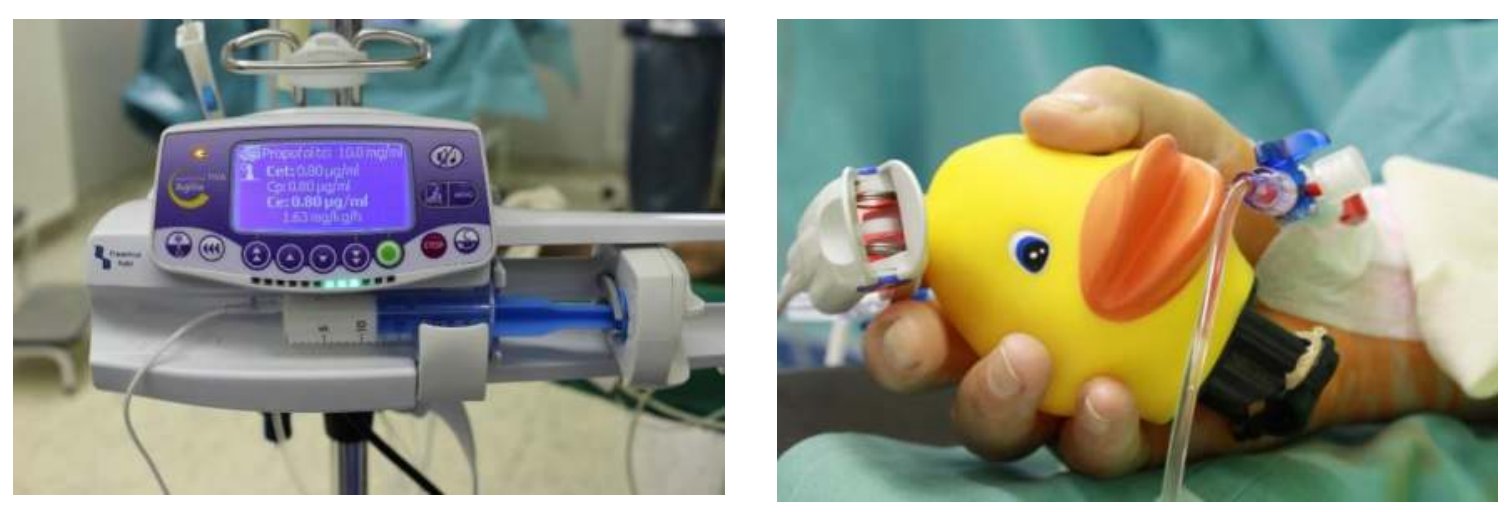

\section{Laboratory measurements:}

Cortisol: prior to surgery (T1), before (T2) and after release of carotid clamp (T3), at 2 (T4) and 24 post-operative hours (T5). Alprazolam: before (T1) and after (T3) the surgery.

\section{Results:}

1. Plasma concentration of cortisol was significantly lower in the TCI group at $\mathrm{T} 2(\mathrm{p}=0.01), \mathrm{T} 3(\mathrm{p}=0.04)$ and $\mathrm{T} 4(\mathrm{p}=0.006)$ than in the BDZ group

2. Alprazolam plasma concentrations did not correlate with cortisol concentrations at any time point

3. Significant positive correlation was found between the clamp time and plasma cortisol concentration at $\mathrm{T} 3$ $(\mathrm{p}=0.01)$ and the degree of contralateral carotid stenosis and plasma cortisol concentration at $\mathrm{T} 3(\mathrm{p}=0.03)$
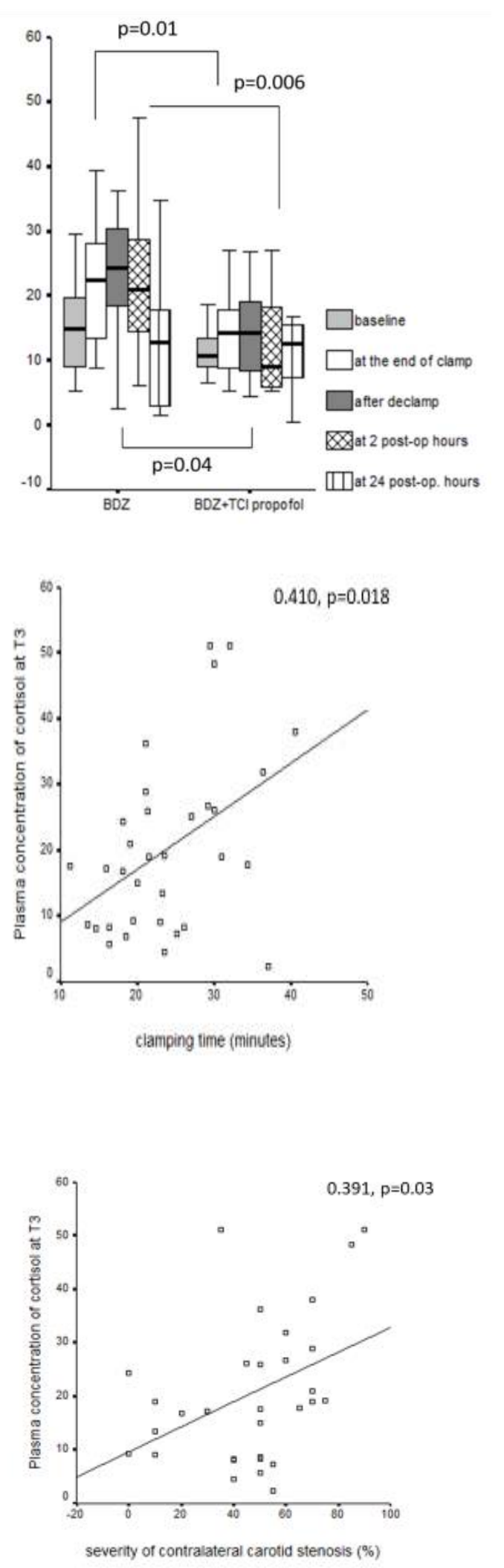

Conclusion: Intraoperative propofol sedation provides better stress relief compared to alprazolam premedication alone during awake CEA.

\section{References:}

- Marrocco-Trischitta MM, Tiezzi A, Svampa MG et al. Perioperative stress response to carotid endarterectomy: the impact of anesthetic modality. J Vasc Surg. 2004; 39:1295-304.

- H. Krenn, E. Deusch, H. Jellinek et al. Remifentanil or propofol for sedation during carotid endarterectomy under cervical plexus block. Br J Anaesth 2002; 89: 637-40 